\title{
Peligrosidad de inundaciones en Extremadura y daños asociados
}

\author{
Flood hazards in Extremadura and associated impacts
}

\author{
Enrique López Rodríguez ${ }^{1^{*}}$ (i) \\ Felipe Leco Berrocal ${ }^{2}$ (D) \\ Ana Beatriz Mateos Rodríguez ${ }^{3}$ (i)
}

\section{Resumen}

Las inundaciones son uno de los riesgos naturales que producen mayores impactos a nivel humano, económico y ambiental, sólo en Europa causaron la pérdida de más de 8.000 vidas en el último siglo. En España los costes económicos asociados a las inundaciones en los últimos 40 años se estiman en más de 5.500.000.000 euros.

El objetivo principal de este trabajo se centra en profundizar en el conocimiento de las inundaciones en Extremadura, tanto desde la óptica de la repercusión espacial de las áreas afectadas como de los impactos asociados a las mismas. El estudio se ha llevado a cabo desde una metodología cualitativa y descriptiva-analítica, utilizando Sistemas de Información Geográfica (SIG) para cartografiar, cuantificar y analizar las variables relacionadas con las inundaciones y con la gestión del riesgo.

Los resultados señalan que en Extremadura la superficie considerada de "alto riesgo" afecta a 30.000 ha y cerca de 19.000 ha se encuentran en situación de "riesgo frecuente", en tanto que el análisis socioeconómico del riesgo muestra que las áreas de regadío concentran la mayor superficie categorizada de "alto riesgo". Se concluye, igualmente, que la cuenca extremeña del Guadiana presenta un mayor riesgo de inundaciones (70\%) que la cuenca del Tajo (30\%).

Palabras clave: inundaciones; cartografía; impacto socioeconómico; Extremadura.

\section{Abstract}

Floods are one of the natural hazards with the greatest human, economic, and environmental impact, causing the loss of more than 8,000 lives in Europe alone in the last century. In Spain, the economic costs associated with floods over the last 40 years are estimated at more than $€ 5.5$ billion.

The main objective of this work is to deepen our knowledge of floods in Extremadura, both from the perspective of the spatial impact of the affected areas and the impacts associated with them. The study has been carried out from a qualitative and descriptive-analytical methodology, using geographic information systems to map, quantify, and analyse the variables related to floods and risk management.

The results indicate that in Extremadura the surface area considered 'high risk' affects 30,000 ha and nearly 19,000 ha are in a situation of 'frequent risk', while the socio-economic analysis of the risk shows that irrigated areas concentrate the largest surface area categorised as 'high risk'. It is also concluded that the Guadiana River Basin in Extremadura has a higher risk of flooding (70\%) than the Tagus River Basin (30\%).

Keywords: floods; mapping; socioeconomic impact; Extremadura.

\footnotetext{
1 Instituto de Investigación en Patrimonio. Universidad de Extremadura. España. enriquelr@unex.es. * Autor para correspondencia.

2 Instituto de Investigación en Patrimonio. Universidad de Extremadura. España. fleco@unex.es

3 Instituto Universitario de Investigación para el Desarrollo Sostenible (INTERRA). Universidad de Extremadura. España. abmateos@unex.es 


\section{Introducción}

En líneas generales, todos los territorios soportan las consecuencias de los riesgos naturales, sobre todo desde el momento en el que los riesgos, de una u otra manera, afectan a la explotación de los recursos naturales, a las actividades humanas o al propio poblamiento (Calvo García-Tornel, 1997). Los riesgos naturales tienen unas consecuencias diferentes en cada territorio afectado, ello depende, por un lado, de la mayor o menor recurrencia del riesgo y de las situaciones de peligro y, por otro lado, del grado de conocimiento en la gestión del riesgo que tenga cada grupo humano en cada sistema territorial (Fernández Garrido, 2006).

Las inundaciones se producen cuando un curso de agua supera el caudal que es capaz de desaguar su canal normal, siendo invadidos los terrenos adyacentes por las aguas y los sedimentos que éstas arrastran (Camarasa Belmonte, 2002). En realidad, el curso de las aguas sigue su camino por el cauce habitual, ocupando en la mayor parte de las ocasiones sus propios lechos inundables o de inundación, por lo que una inundación supone un riesgo en el momento en el que el ser humano ha ocupado dichos lechos, de ahí la consideración de riesgo mixto en múltiples ocasiones (Ayala Carcedo y Olcina Cantos, 2002) ante la ocurrencia del fenómeno de las inundaciones.

Las inundaciones son un proceso en el que juega un papel importante el umbral de tolerancia al exceso de agua, es por ello que la relación ser humano-medio en torno al agua, presenta dos facetas diferenciadas, una de recurso, y otra de riesgo; y ambas están incluidas en el umbral del grado de desarrollo de las comunidades humanas, por lo que presentarán variabilidad espacial y temporal.

Una vez iniciada la secuencia de precipitación, inmediatamente, el agua pasa a formar parte del caudal de los cursos fluviales. Es por ello imprescindible conocer las características de las diferentes cuencas para evitar, en la medida de lo posible, la generación de riesgo por inundaciones atendiendo fundamentalmente a las características tipológicas, morfológicas, grado de pendiente, litología e incluso el estado de saturación del suelo en un momento dado. En base a estas características, las cuencas y subcuencas de Extremadura, delimitadas en el espacio de las cuencas interiores de la península (Demarcación Hidrográfica del Tajo y del Guadiana eminentemente), se clasifican como las menos torrenciales al tratarse de cuencas alargadas, de baja pendiente y largo recorrido.

Según los datos publicados por EM-DAT (The International Disaster Database), y en función de los parámetros de frecuencia, impacto social e impacto económico, las inundaciones suponen el riesgo con mayor número de siniestros y mayor coste económico asociado para el conjunto europeo (UE28). En este sentido, las cifras para el periodo 1905-2016 es de 8.023 muertes y un coste económico de 121.094.000.000 dólares. En el caso español, los datos ofrecidos por la Dirección General de Protección Civil (2016), cifra en 329 las muertes producidas por las inundaciones en el periodo 1995-2015, constituyendo la principal causa de muerte por riesgo natural en España.

En términos económicos, los datos desagregados del Consorcio de Compensación de Seguros (CCS) reflejan que las inundaciones son la causa natural de mayor impacto en cuanto a daño a los bienes, con un total de 551.432 expedientes y un coste de 5.564.323.446 €, lo que supone el 61,3\% del total de causas extraordinarias para el periodo 1971-2015. Por su parte, en relación con los daños ocasionados a las personas, las inundaciones han supuesto un coste de 5.350.695 €, ocupando el segundo lugar por daño a las personas en el mismo periodo.

Destaca el hecho de que, a diferencia de otro tipo de riesgos, las inundaciones determinan una considerable fuerza dinámica del proceso de cambio del medio ambiente, y existe una marcada incertidumbre motivada por la correlación entre su escala y sus consecuencias (EEA, 1998). Uno de los mejores ejemplos de actuación sectorial de la Unión Europea en materia de riesgos, fue la aprobación de la Directiva 2007/60/CE del Parlamento Europeo y del Consejo, de 23 de octubre de 2007, relativa a la evaluación y gestión de los riesgos de inundación. Este documento tiene como fin último el establecimiento de un marco para la evaluación y gestión de los riesgos de inundación, donde específicamente se compromete a la reducción del impacto negativo no sólo sobre la población o el medio ambiente sino también sobre el patrimonio cultural y las actividades socioeconómicas sobre las que devengue el fenómeno.

Los instrumentos de ordenación del territorio se constituyen como una herramienta de defensa eficaz con la capacidad de adecuar los usos del suelo a las necesidades y características del medio físico y su naturaleza fenomenológica. Además, como herramienta, permite la gestión integrada del riesgo condicionando el desarrollo de usos del suelo localizados en áreas susceptibles de inundación (López, 2015). 
Una de las formas más rigurosas de estudio para entender la configuración del territorio, orientada a la comprensión de las dinámicas relacionadas con los procesos de inundaciones, lo constituye la cartografía de zonas inundables. Este tipo de cartografía ha de ser regulada y facilitada de manera oficial por la administración competente. En el contexto europeo, dadas las importantes implicaciones socioeconómicas de las inundaciones, existe una importante cantidad y calidad de la información cartográfica disponible. La cartografía asociada al riesgo de inundaciones suele representar la extensión potencial y profundidad las inundaciones, elementales para la planificación espacial, así como para la estimación de los daños por inundación (Van Alphen, Martini, Loat, Slomp y Passchier, 2009). En el caso español, se trata del Ministerio para la Transición Ecológica, amparado bajo el auspicio del Real Decreto 903/2010, de 9 de julio, de evaluación y gestión de riesgos de inundación (B.O.E. núm. 171, de 15 de julio de 2010), que traspone la Directiva 2007/60/CE del Parlamento Europeo y del Consejo, de 23 de octubre, relativa a la evaluación y gestión de los riesgos de inundación (D.O.U.E., núm. 288, de 6 de noviembre de 2007).

Los trabajos de investigación sobre riesgos naturales en Extremadura tienen un marcado carácter sectorial, así pueden encontrarse varias publicaciones centradas en determinados fenómenos, caso de los incendios forestales por su marcado índice de siniestralidad en la región (García Lucas y Carrascal Tirado, 2007; Blas Morato y Nieto Masot, 2008; Blas Morato, 2009; Moya et al., 2009; Mateos Rodríguez, Leco Berrocal y López Rodríguez, 2015; Navazo Arenas, Nieto Masot y Moreno Marcos, 2016; Pulido Díaz, Bertomeu García, del Pozo Barrón, Giménez Fernández y Moreno Marcos, 2017). Hay que añadir además que la mayoría de estos trabajos se centran en el campo de la legislación, planificación, detección y prevención, siendo muy pocos los trabajos que se centran en aspectos tales como el impacto social (López Rodríguez, 2017).

Desde el punto de vista de la comprensión integrada y del estudio de los fenómenos naturales para el conjunto territorial extremeño, sólo unas pocas publicaciones han tratado de ofrecer una visión de conjunto que permitan comprender la distribución espacial del fenómeno en Extremadura. Este aspecto es fundamental dadas las altas implicaciones del campo de los riesgos naturales en materia como la ordenación territorial, siendo ésta abordada y legislada desde la administración autonómica. Sin duda, el caso más paradigmático que engloba todos y cada uno de los riesgos de la región lo constituye el propio Plan Territorial de Protección Civil de la Comunidad Autónoma de Extremadura (PLATERCAEX) (Junta de Extremadura, 2006).

Por otro lado, desde el punto de vista científico existen trabajos orientados a otorgar visibilidad a los fenómenos o a herramientas para su estudio, análisis y cuantificación (Nieto Masot, García Paredes y Fernández Sánchez, 2011; López Rodríguez, 2015; Mateos Rodríguez y López Rodríguez, 2016, López Rodríguez, 2019).

Por todo ello la presente investigación tiene como objetivo entender cómo se produce el fenómeno de las inundaciones en la Comunidad Autónoma de Extremadura, haciendo especial hincapié en el análisis de los espacios inundables, categorizados como zonas de riesgo potencial y su caracterización territorial; en el estudio del impacto socioeconómico, en términos de costes económicos y población potencialmente expuesta; así como sobre los usos del suelo.

\section{Metodología}

\section{1. Área de Estudio}

La Comunidad Autónoma de Extremadura se localiza al suroeste de la Península Ibérica, limitando al oeste con Portugal, al este con la Comunidad Autónoma de Castilla La Mancha, al norte con la Comunidad Autónoma de Castilla y León y al sur con la Comunidad Autónoma de Andalucía. Extremadura se conforma por las provincias de Cáceres y Badajoz, siendo éstas de las de mayor superficie de España, y aunando un total de $41.633 \mathrm{~km}^{2}$. Cuenta con una población de 1.065 .000 habitantes, con una densidad demográfica ciertamente escasa, en torno a 26,4 habitantes $/ \mathrm{km}^{2}$.

En Extremadura encontramos hasta cuatro demarcaciones hidrográficas: Tajo, Guadiana, Guadalquivir y un pequeño ámbito territorial de la del Duero. No obstante, podemos afirmar que se encuentra integrada esencialmente por las cuencas hidrográficas del Tajo y del Guadiana, conformadas por una importante red de cauces menores y afluentes de los principales ríos. Así, en el río Tajo discurre encajado por la penillanura trujillano-cacereña, destacando importantes afluentes como el Tiétar, Alagón, Jerte, Almonte o Salor. Por su parte, el río Guadiana se caracteriza por una mayor extensión y amplitud en su cuenca, la cual discurre por terrenos de vega en llanura óptimos para el aprovechamiento agrícola bajo explotaciones en regadío. 
Figura 1. Localización del área de estudio, y principales elementos hidrográficos

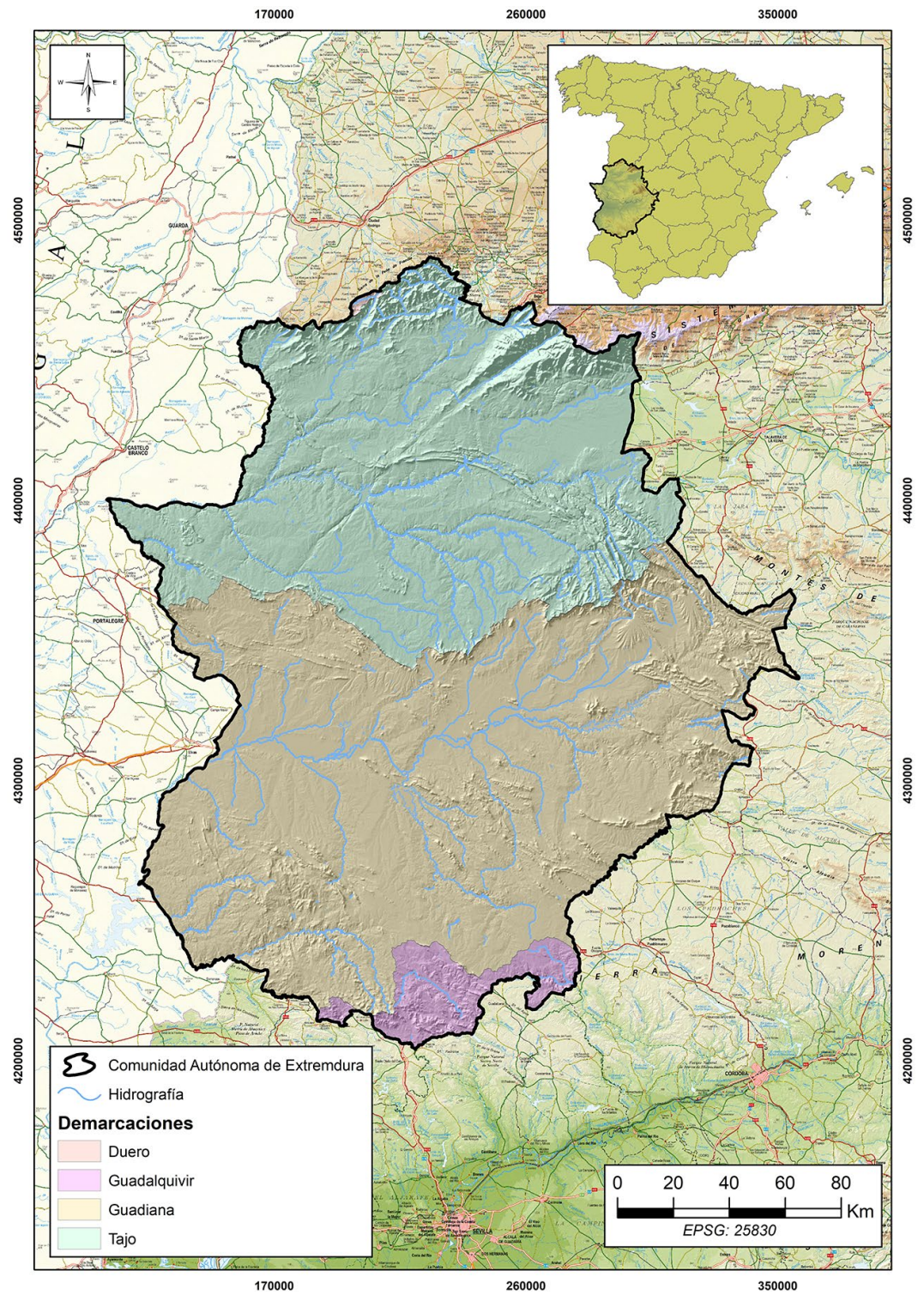

Fuente: Instituto Geográfico Nacional (IGN). Elaboración propia

\subsection{Las inundaciones en Extremadura}

Respecto a las inundaciones producidas en las cuencas extremeñas, según la Tesis Doctoral realizada por la investigadora Potenciano de las Heras (2004), para las inundaciones históricas ocurridas en la cuenca del Tajo se determina que al menos el $73 \%$ se han producido en la parte alta de la misma, mientras que la parte baja se ve afectada por tan sólo un $9 \%$ de las inundaciones, quedando el resto repartido por toda la cuenca. La distribución estacional de las mismas se corresponde con un máximo en invierno (58\%), coincidente con el máximo pluviométrico, y un segundo máximo en otoño (20\%). La magnitud de las inundaciones del Tajo es elevada, tal es así que un $21 \%$ de las mismas es de magnitud extrema, siendo las precipitaciones abundantes su causa principal (29\%).

Por otra parte, para la cuenca del Guadiana el registro histórico señala que, tanto la parte baja (42\%) como la parte alta (36\%), se ven afectadas normalmente por la recurrencia de las inundaciones. En este sentido, la distribución estacional de los eventos indica, igual que en el caso anterior, el predominio de los registros ocurridos en invierno (53\%), frente a un $23 \%$ de registros en otoño. Igualmente, la mayor parte de los eventos se debe a lluvias torrenciales y tormentas ( $70 \%$ sobre el total) (Potenciano de las Heras, 2004). 
Tabla 1. Clasificación de las inundaciones históricas en las cuencas del Tajo y Guadiana (en \%)

\begin{tabular}{|c|c|c|c|}
\hline \multirow{2}{*}{\multicolumn{2}{|c|}{ Variables de la clasificación }} & \multicolumn{2}{|c|}{ Cuencas } \\
\hline & & \multirow{2}{*}{\begin{tabular}{|l} 
Tajo \\
73
\end{tabular}} & \multirow{2}{*}{$\begin{array}{r}\text { Guadiana } \\
36\end{array}$} \\
\hline \multirow{3}{*}{ Distribución espacial } & Cuenca alta & & \\
\hline & Cuenca baja & 9 & 42 \\
\hline & Toda la cuenca & 18 & 22 \\
\hline \multirow{4}{*}{ Distribución estacional } & Otoño & 20 & 23 \\
\hline & Invierno & 58 & 53 \\
\hline & Primavera & 12 & 9 \\
\hline & Verano & 10 & 15 \\
\hline \multirow{3}{*}{ Magnitud } & Extrema & 21 & 10 \\
\hline & Alta & 57 & 48 \\
\hline & Media & 22 & 42 \\
\hline \multirow{5}{*}{ Causas } & Lluvia & 29 & 56 \\
\hline & Deshielo & 4 & 1 \\
\hline & Tormenta & 8 & 14 \\
\hline & Estructural & 2 & 5 \\
\hline & Marea & - & 4 \\
\hline \multirow{3}{*}{ Tipos } & Crecida con desbordamiento & 51 & 31 \\
\hline & Avenida & 34 & 38 \\
\hline & Estancamientos & 15 & 31 \\
\hline
\end{tabular}

Fuente: Potenciano de las Heras, 2004

El Plan Especial ante el Riesgo de Inundaciones de la Comunidad Autónoma de Extremadura (INUNCAEX) recoge un total de 136 eventos de inundación registrados en este territorio en el periodo 19201999. La cuenca del Guadiana registra un $70,6 \%$ de los eventos, frente a un 30\% de la cuenca del Tajo que, sin embargo, registra en el entorno del Sistema Central el 65\% de todos los eventos ocurridos en esta cuenca, siendo las áreas Gredos, Plasencia y Coria aquellas que tienen mayor índice de concentración.

Para el caso del río Guadiana, el mayor riesgo se centra en la zona de las Vegas (Altas y Bajas) (Juárez Sánchez-Rubio, 1978), donde se han registrado numerosas inundaciones por desbordamiento de ríos como el Ruecas, Gargáligas, Zújar y Guadiana (López Rodríguez, 2015).

\subsection{Materiales}

El presente estudio parte de la recopilación de datos e información disponible, no sólo a través de la variada producción científica relativa al fenómeno de las inundaciones, sino también a partir del análisis de las fuentes procedentes de marcos normativos y disposiciones legales, como son la planificación de Protección Civil en materia de emergencias, y más concretamente, de los fenómenos de inundación, así como los datos estadísticos provenientes del Consorcio de Compensación de Seguros (CCS).

Para la realización de los análisis cartográficos, se han tomado como referencia las coberturas disponibles de superficies inundables por período de retorno o recurrencia del MITERD.

Entre los servicios disponibles en materia de cartografía de zonas inundables por período de recurrencia (10, 50, 100 y 500 años), se han tomado para el análisis, tanto la superficie, como la relación con las actividades económicas afectadas, así como el riesgo a la población, las correspondientes a Alta Probabilidad o Alto Riesgo (10 años), Media Probabilidad o Riesgo Frecuente (50 años), y Probabilidad media u ocasional (100 años).

Para el análisis relativo a la ocupación del suelo, se ha tenido como referencia fundamental el Sistema de Información de Ocupación del Suelo de España (SIOSE) de la Comunidad Autónoma de Extremadura, proveniente del Centro de Descargas del Centro Nacional de Información Geográfica del IGN.

\subsection{Metodología}

La presente investigación se ha basado en la extracción de los datos y coberturas cartográficas disponibles, a partir de la cartografía del Sistema Nacional de Cartografía de Zonas Inundables, y la explotación 
de sus datos asociados y relación con otros conjuntos de datos espaciales, así como la inclusión de información estadística relativa a los impactos producidos en el territorio.

Los correspondientes procedimientos cartográficos se han basado en la adecuación geométrica, a base de técnicas de recorte, intersección y áreas de influencia de las coberturas de zonas inundables del MITERD con la delimitación territorial de Extremadura.

Para el análisis de la ocupación del suelo en las zonas inundables, se han utilizado, por un lado las coberturas obtenidas a partir de la integración de las zonas de riesgo con los usos y cubiertas del suelo del SIOSE, ya que, a través de esta integración, pueden determinarse las características territoriales intrínsecas de las distintas áreas potencialmente expuestas. Y por otro lado, mediante la integración de los datos del MITERD relativos a las Áreas de Riesgo Potencial Significativo de Inundación (ARPSI), y concretamente a la información del riesgo a las actividades económicas, la cual incluye la información relativa a la estimación económica por usos del suelo en las distintas áreas de riesgo, por periodo de recurrencia, de los que se ha empleado los periodos de 10 y 100 años.

El cálculo de la población estimada en las zonas inundables efectuado por el MITERD se lleva a cabo mediante la integración de diversas fuentes de información. El objetivo fundamental es poder representar la afección a la población mediante las zonas inundables, donde además se le añaden los atributos de población estimada en la zona afectada para cada término municipal y la población total por término municipal. Según las reseñas metodológicas analizadas, este proceso se basa en la superposición de la envolvente del período de retorno correspondiente a cada término municipal afectado con la información espacial de densidad de población procedente de información geográfica de referencia ${ }^{4}$.

El análisis y tratamiento cartográfico ha sido realizado mediante el uso de Sistemas de Información Geográfica, en concreto se ha utilizado el software ArcGIS, así como el empleo de los paquetes estadísticos de Microsoft Excel.

\section{Resultados}

\subsection{Espacios inundables y riesgo de inundación en Extremadura}

En Extremadura existe una importante superficie potencialmente afectable por el fenómeno de la inundación. Si bien, al comparar los datos entre las dos grandes demarcaciones hidrográficas, puede apreciarse una clara dicotomía entre la superficie contenida en el Tajo y el Guadiana. En este sentido, se han contabilizado un total de 27.530,10 ha de zonas de "alto riesgo" en el Guadiana frente a las 3.878,12 ha en el Tajo. En el caso de la cuenca extremeña del Guadiana esa superficie supone el 87,65\% de la superficie afectable total, frente al 12,35\% que representa la del Tajo. El total de superficie de "alto riesgo" en Extremadura se cifra en $31.408,22$ ha entre ambas demarcaciones.

Tabla 2. Relación de la superficie de las zonas inundables por tiempo de recurrencia y demarcación

\begin{tabular}{|l|r|r|l|r|r|}
\hline \multicolumn{3}{|c|}{ Alto Riesgo ( $\mathrm{T}=10$ años) } & \multicolumn{3}{c|}{ Riesgo Frecuente ( $\mathrm{T}=50$ años) } \\
\hline Demarcación & Superficie $(\mathrm{ha})$ & $\%$ & Demarcación & Superficie (ha) & \multicolumn{1}{c|}{$\%$} \\
\hline Guadiana & $27.530,10$ & 87,65 & Guadiana & $17.427,08$ & 92,50 \\
\hline Tajo & $3.878,12$ & 12,35 & Tajo & $1.412,38$ & 7,50 \\
\hline Total general & $31.408,22$ & 100,00 & Total general & $18.839,46$ & 100,00 \\
\hline
\end{tabular}

Fuente: MITERD. Elaboración propia

Otro ejemplo que evidencia la diferenciación entre ambas demarcaciones es el relativo a las cifras obtenidas para las zonas de "riesgo frecuente" ( $\mathrm{T}=50$ años), donde aun existiendo menor superficie potencial de riesgo (18.839,46 ha, un 25,01\% menos con respecto a las zonas de "alto riesgo"), se observa una fuerte concentración en la Demarcación del Guadiana, representando ésta el 92,5\% de la superficie potencialmente afectable como "riesgo frecuente", alcanzando un total de 17.427,08 ha.

\footnotetext{
4 Las fuentes empleadas para la estimación de la densidad de población se basan en: un fichero ráster (imagen) cuyo tamaño de píxel es de 100x100 metros de EUROSTAT, las Bases de Datos de Poblaciones, así como Ortofotos del IGN, y la información disponible de la D.G. del Catastro.
} 
Figura 2. Detalle de la zonificación de alto riesgo ( $\mathrm{T}=10$ años) en las Vegas Bajas del Guadiana

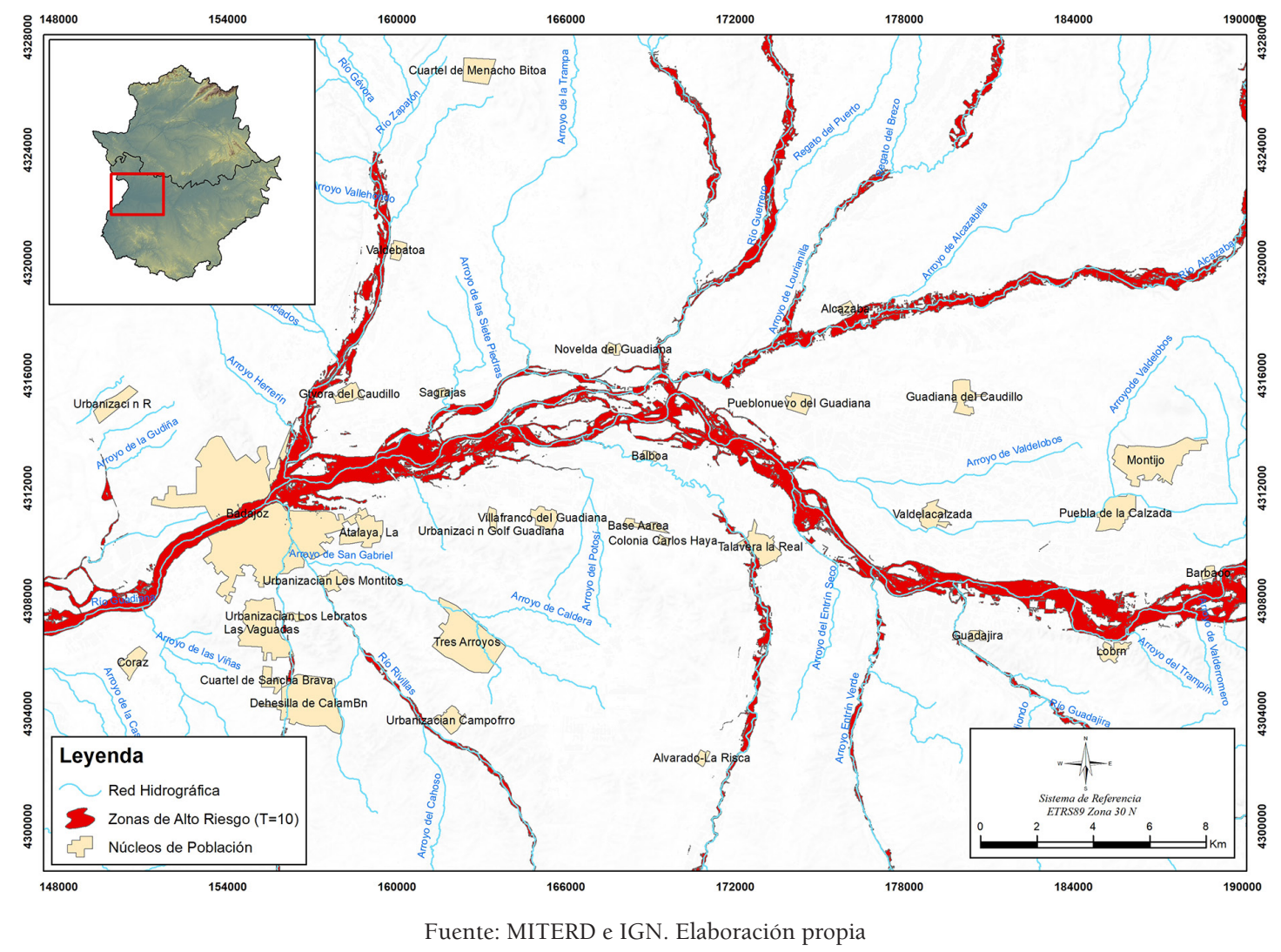

Figura 3. Detalle de la zonificación de alto riesgo ( $T=10$ años) en las Vegas Altas del Guadiana

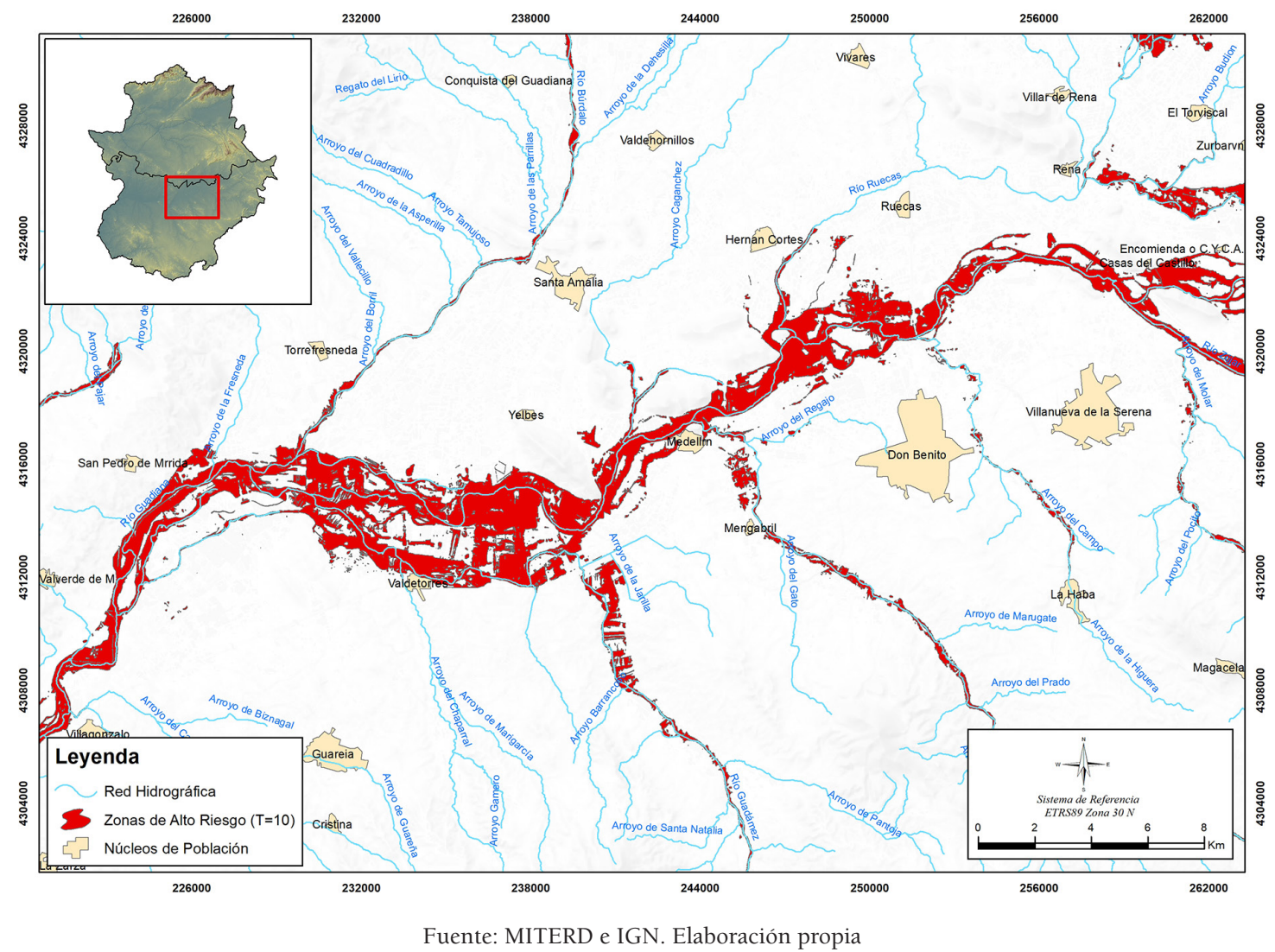


Figura 4. Detalle de la zonificación de riesgo frecuente ( $T=50$ años) en las Vegas Bajas del Guadiana

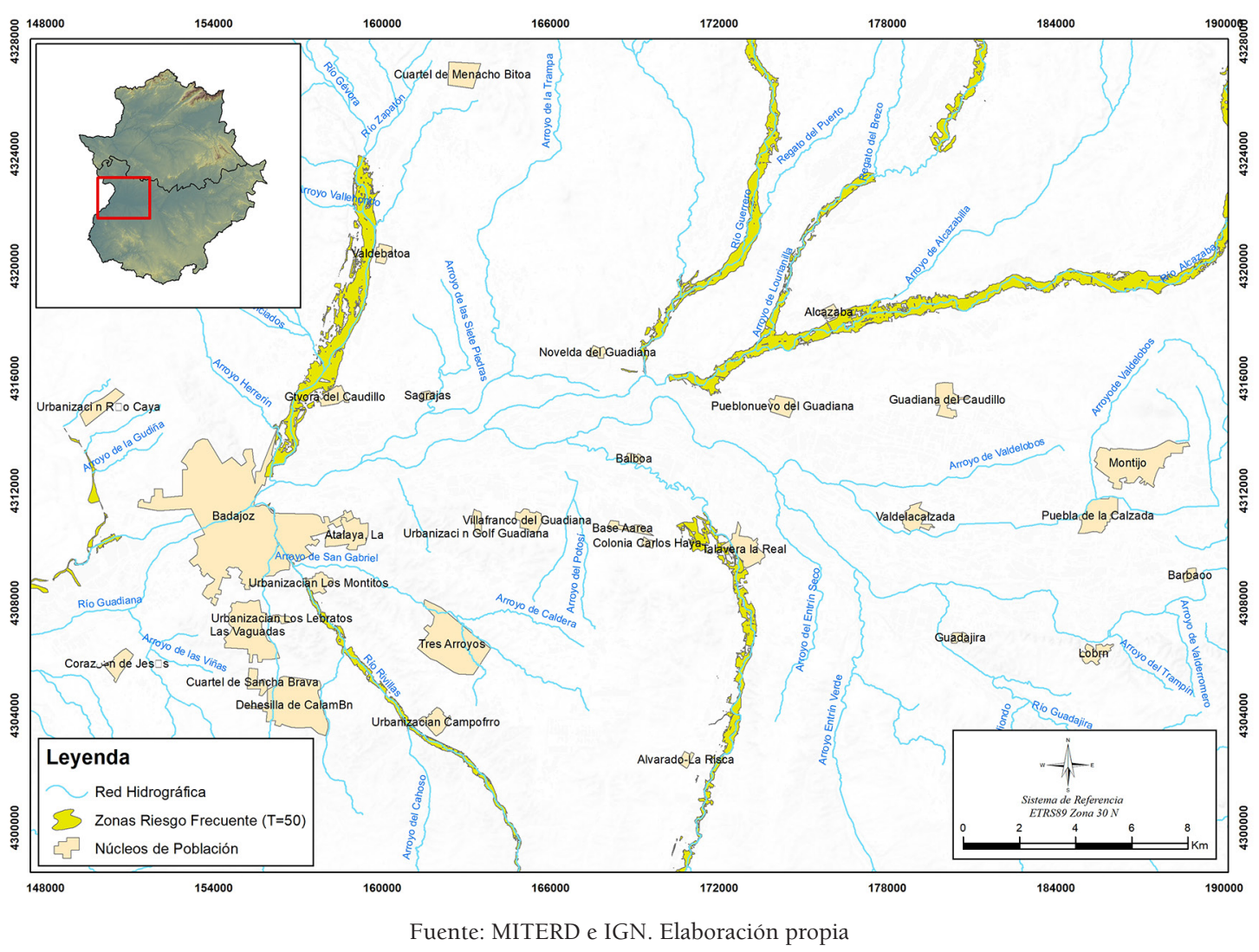

Figura 5. Detalle de la zonificación de riesgo frecuente ( $T=50$ años) en las Vegas Altas del Guadiana

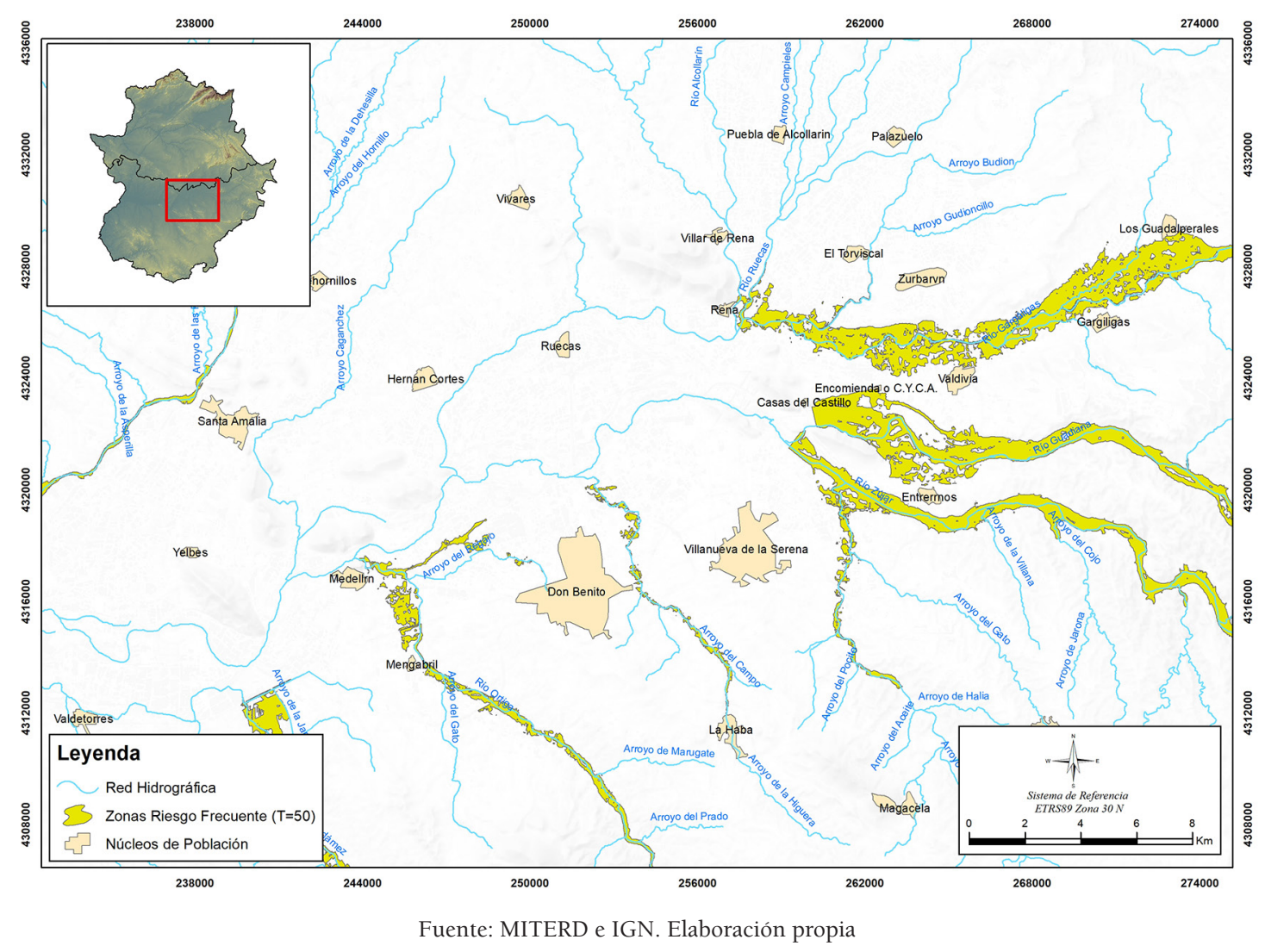




\subsection{Usos del suelo e impactos económicos asociados en zonas de alto riesgo}

Los datos relativos a las zonas de alto riesgo muestran un claro predominio de la actividad agraria en regadío, una de las más importantes en términos de desarrollo socioeconómico para la región. Hasta 7.638 ha bajo esta forma de aprovechamiento se encuentran en zonas de "alto riesgo" de inundación, lo que supone el $57,15 \%$ del total de superficie estimada. Los daños económicos, a su vez, son los de mayor alcance con un total estimado para 10 años de 243.771.228 €, lo que supone casi la mitad del daño total (el 42,9\%).

En términos de superficie, el segundo gran grupo afectado serían los usos forestales (donde se incluyen gran variedad de cubiertas y usos del suelo) con 2.572 ha, no obstante, el impacto económico de esta superficie tan sólo supone el $0,2 \%$. La actividad económica con mayores daños asociados tras la agricultura de regadío son las infraestructuras hidráulicas, cuyos costes ascenderían a $110.411 .305 €$, el $19,4 \%$ del coste total. Por este motivo, es importante conocer que no existe una relación directa entre la superficie expuesta con el daño económico asociado al posible impacto, ya que, en este último caso, este tipo de actividad económica sólo representa el 0,23\% de la superficie estimada, poco más de 30 has. El tercer lugar en daños económicos estimados lo ocupan las carreteras, las cuales, pese a ocupar una superficie de 48,3\% en zona de "alto riesgo", su coste económico se eleva a la cifra de $71.291 .762 €$, un 12,5\% del total de daño estimado.

En términos de cómputos globales por actividad económica, se estima un total de 13.365,7 ha afectadas relevantes en zonas de "alto riesgo" de inundación, siendo los costes económicos totales calculados para 10 años de 568.203.838 €. A continuación se muestra la relación de actividades económicas, superficie y daño económico para distintos usos del suelo con períodos de retorno de inundaciones de 10 años, lo que supone a la zonificación de alto riesgo de inundaciones:

Tabla 3. Estadísticas de las actividades económicas, superficies afectadas y daños económicos estimados en Extremadura (Período de retorno $=10$ años)

\begin{tabular}{|c|c|c|c|c|}
\hline \multirow{2}{*}{ Tipo de Actividad Económica } & \multicolumn{2}{|c|}{ Superficie } & \multicolumn{2}{|c|}{ Daños económicos estimados } \\
\hline & $\mathrm{Ha}$ & $\%$ & Euros & $\%$ \\
\hline Agrícola-Regadío & $7.638,3$ & 57,15 & 243.771 .228 & 42,9 \\
\hline Infraestructuras hidráulico-sanitarias & 30,5 & 0,23 & 110.411 .305 & 19,4 \\
\hline Infraestructuras: carreteras & 48,3 & 0,36 & 71.291 .762 & 12,5 \\
\hline Infraestructuras: residuos & 24,9 & 0,19 & 36.711 .660 & 6,5 \\
\hline Infraestructura social & 38,8 & 0,29 & 20.444 .068 & 3,6 \\
\hline Industrial concentrado & 3,6 & 0,03 & 12.880 .170 & 2,3 \\
\hline Asociado a urbano & 35,1 & 0,26 & 11.973 .738 & 2,1 \\
\hline Otros usos rurales & $2.490,0$ & 18,63 & 10.635 .008 & 1,9 \\
\hline Urbano concentrado & 5,4 & 0,04 & 9.548 .560 & 1,7 \\
\hline Edificación asociada a urbano disperso & 6,6 & 0,05 & 8.839 .759 & 1,6 \\
\hline Industrial disperso & 7,8 & 0,06 & 8.701 .569 & 1,5 \\
\hline Edificación asociada a urbano concentrado & 3,3 & 0,02 & 8.368 .847 & 1,5 \\
\hline Edificación asociada a industrial disperso & 1,8 & 0,01 & 5.869 .724 & 1,0 \\
\hline Infraestructuras: ferrocarriles & 2,5 & 0,02 & 4.185 .808 & 0,7 \\
\hline Agrícola-Secano & 328,1 & 2,45 & 2.055 .582 & 0,4 \\
\hline Terciario & 1,7 & 0,01 & 1.403 .923 & 0,2 \\
\hline Forestal & $2.572,1$ & 19,24 & 885.862 & 0,2 \\
\hline $\begin{array}{l}\text { Edificación asociada a industrial concen- } \\
\text { trado }\end{array}$ & 0,1 & 0,00 & 98.383 & 0,0 \\
\hline Urbano disperso & 0,1 & 0,00 & 80.682 & 0,0 \\
\hline Otras áreas sin riesgo & 126,8 & 0,95 & 46.200 & 0,0 \\
\hline Total general & $13.365,7$ & 100 & 568.203 .838 & 100 \\
\hline
\end{tabular}

Elaboración propia 
Figura 6. Relación de las actividades y usos del suelo con representación de al menos un 2\% del daño económico estimado por inundación en zonas de Alto Riesgo ( $\mathrm{T}=10$ años)

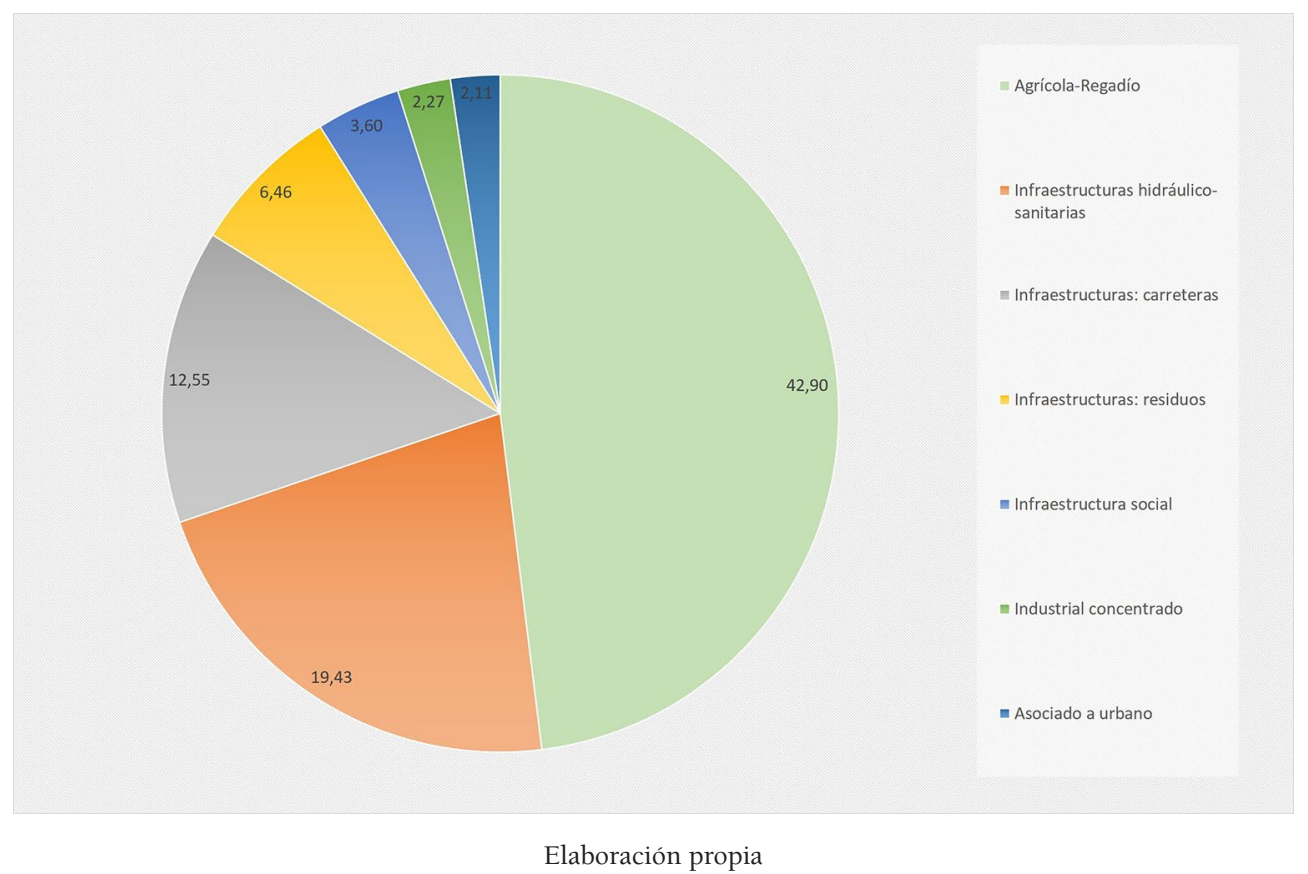

Figura 7. Datos del importe estimado por actividad económica y uso del suelo en zonas de alto riesgo de inundación con representación de al menos un $1 \%$ del total

300000000

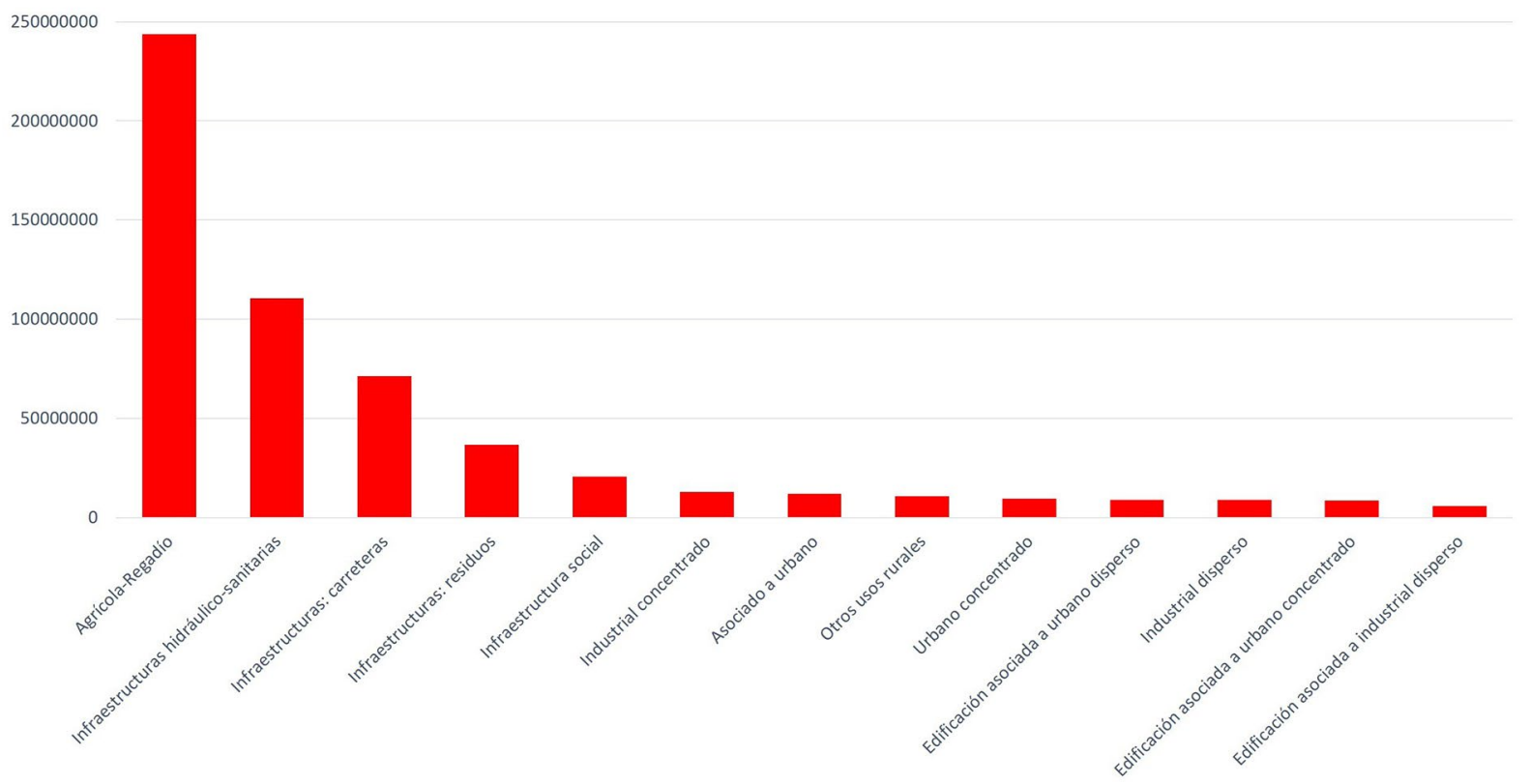

Elaboración propia

Complementando el análisis de los datos económicos, cabe apuntar el hecho de que en la serie estadística disponible del Consorcio de Compensación de Seguros (CCS) se expresa el dato producido por las graves inundaciones ocurridas en Badajoz en 1997 (inundaciones y tempestad ciclónica atípica). El CCS apunta un total de 3.003 reclamaciones producidas, elevándose el coste de las indemnizaciones a un total de 27.489.110 € (CCS, 2016). 
Figura 8. Actividades económicas en zonas de alto riesgo (10 años) en Badajoz

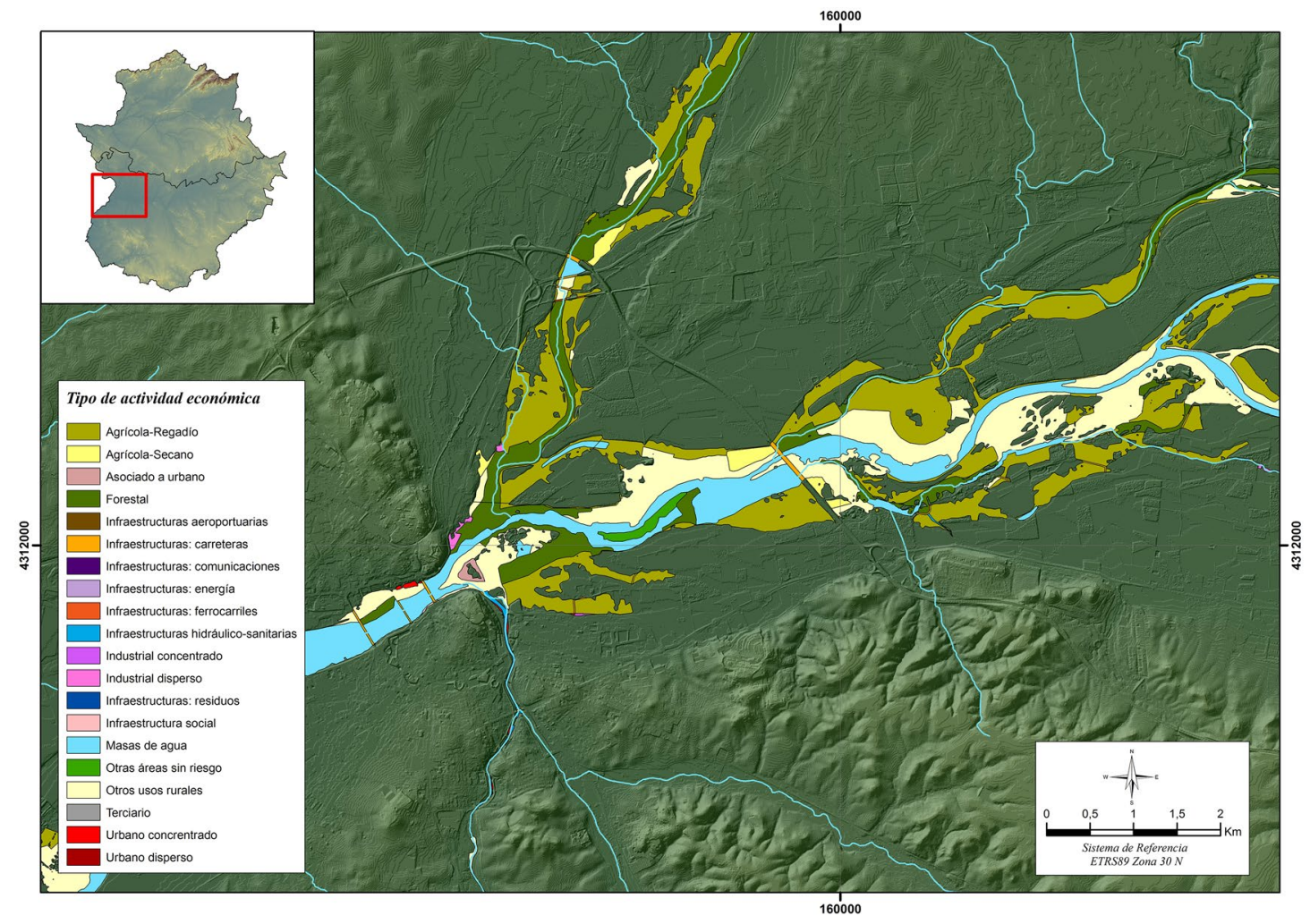

Fuente: MITERD e IGN. Elaboración propia

\subsection{Impacto social de las inundaciones}

Otros de los aspectos más relevantes, derivados del análisis cartográfico de los servicios del MITERD, es la estimación de la población afectada por inundación, bien sea a 10 años o 100 años de recurrencia. En este sentido, se han tomado las coberturas cartográficas disponibles para la extracción de la información alfanumérica correspondiente:

Tabla 4. Número de habitantes estimados en zonas inundables por período de recurrencia

\begin{tabular}{|c|c|c|}
\hline \multirow{2}{*}{ Provincias } & $\mathrm{T}=10$ años & $\mathrm{T}=100$ años \\
\hline & $N^{o}$ Habitantes Zona Inundable & $N^{o}$ Habitantes Zona Inundable \\
\hline Cáceres & 3.211 & 6.144 \\
\hline Badajoz & 3.515 & 14.074 \\
\hline Total & 6.726 & 20.218 \\
\hline
\end{tabular}

Elaboración propia

Asimismo, y dado que los datos aportados poseen un carácter espacial, no sólo con la envolvente de las propias zonas inundables, sino también con la referencia municipal al que pertenece a través del código INE, se ha podido presentar la información de forma estadística por términos municipales, presentando para toda Extremadura la siguiente distribución:

Los datos extraídos reflejan que, al menos, un total de 6.726 personas se ubican en las zonas de "alto riesgo" de inundación, cuya distribución es bastante homogénea entre la provincia de Cáceres, con 3.211 habitantes, y la provincia de Badajoz, con 3.515 habitantes. Por su parte, las estadísticas extraídas para el tiempo de recurrencia de 100 años presentan unas cifras mayores, pasando la provincia de Cáceres a 6.144 habitantes frente a 14.074 habitantes en la provincia de Badajoz, lo que supone un importante aumento de la población potencialmente expuesta al riesgo de inundación. 
Figura 9. Distribución del número de habitantes en zonas de "alto riesgo" de inundación por término municipal

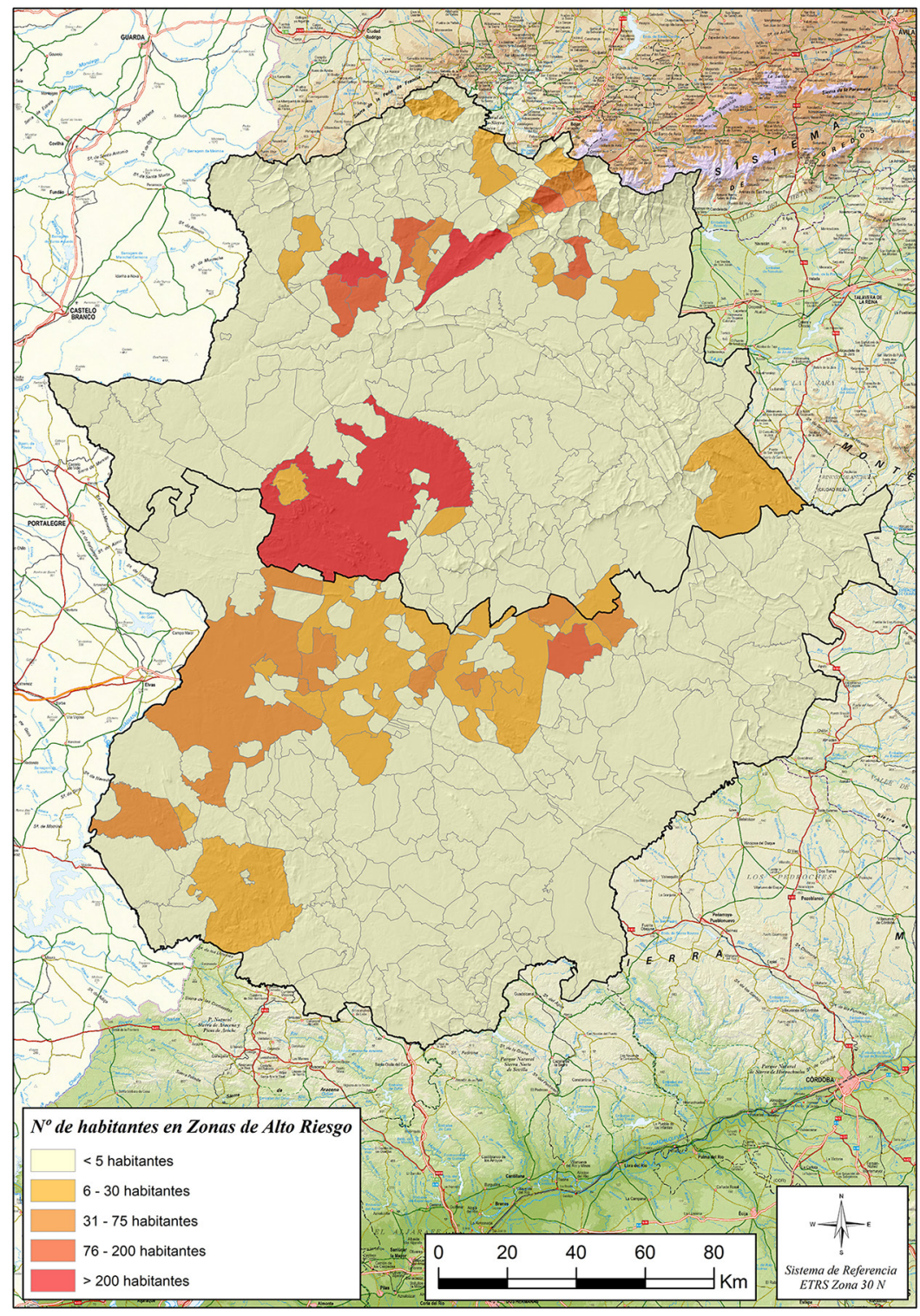

Elaboración propia

\section{Discusión}

Parece evidente que existen regiones-riesgo clasificadas y registradas en el planeta, en las cuales los fenómenos naturales de carácter extraordinario se producen con mayor asiduidad que en el resto (Calvo García-Tornel, 1997; Ayala Carcedo y Olcina Cantos, 2002; Saurí i Pujol y Ribas Palom, 2006; Olcina Cantos, 2008). Pero los fenómenos naturales no entienden de fronteras o regiones, y cualquier territorio es susceptible de sufrir un episodio de carácter extraordinario que induzca a una catástrofe. Entre ellos encontramos regiones como Extremadura, donde se ha constatado el hecho de que las inundaciones constituyen un tipo de fenómeno de riesgo con potencial para generar impactos socioeconómicos asociados de gran alcance.

Pese a la importante profusión de trabajos relacionados con otros fenómenos de riesgo como los incendios forestales en Extremadura, existen muy pocos trabajos que aporten visibilidad a fenómenos como las inundaciones, y más teniendo en cuenta que éstas se producen con mayor asiduidad en las zonas agrícolas bajo formas de explotación en regadío, una de las actividades de mayor peso productivo y económico en la región. 
Incluir análisis de riesgos en los procesos de planificación y gestión del territorio permite introducir una serie de variables que refuerzan la capacidad de los territorios de hacer frente a las posibles consecuencias de los efectos adversos de la naturaleza. Este tipo de análisis posibilitará la identificación de amenazas, el umbral crítico de cada territorio, el diseño de alternativas, las medidas de reducción, la aplicabilidad de marcos normativos e institucionales, así como los elementos y factores a tener en cuenta tras la aplicación de cualquier medida de planificación sobre el territorio (Patricia Torchia, 2011).

La vulnerabilidad territorial es un concepto amplio y difuso (Ruiz Pérez, 2012). A su vez, el nivel de implicación social que las personas poseen con su entorno, y cómo se gestionan, repercute directamente en los niveles de percepción del propio riesgo (Saurí i Pujol y Ribas Palom, 2006; Saurí, Ribas, Lara y Pavón, 2010). El desarrollo de acciones de carácter educacional sobre la población, y más si ésta es susceptible a sufrir las consecuencias o impactos de los riesgos naturales, produce importantes mejoras sobre la dimensión cultural del propio riesgo (Morote Seguido y Pérez Morales, 2019; López Martínez, Pérez Morales, y Gil Guirado, 2016).

La determinación de la vulnerabilidad social se relaciona fuertemente con los determinantes socioeconómicos y demográficos, pudiéndose analizar de forma multinivel desde la distribución geográfica hasta la variabilidad espacial (Frigerio y De Amicis, 2016). Esto se relaciona también con los procesos de enfoque participativo, como el desarrollado por Bustillos Ardaya, Evers y Ribbe (2019), donde a partir de técnicas basadas en la cartografía colaborativa, promueve la participación, facilita los procesos de comunicación y aprendizaje social y crea material confiable, cuantitativo y útil para la comparación y toma de decisiones; aspectos que influye de forma notoria en la evaluación y gestión de riesgos y la determinación de la gobernanza.

Gracias al avance en las Tecnologías de Información Geográfica (TIG), los análisis de riesgos pueden sucederse a una escala y complejidad cada vez mayores, permitiendo el avance en los procesos de gestión territorial, así como la extracción de conclusiones aplicables a las distintas escalas administrativas, ya sean locales o regionales (Gallegos Reina y Perles Roselló, 2019). La cartografía de riesgos se constituye como la principal herramienta orientada a la prevención, reducción y mitigación en riesgos como las inundaciones, debiendo mantener a su vez un importante papel en todo proceso de toma de decisión, teniendo en cuenta además las tendencias hacia el incremento de intensidad de los fenómenos en el contexto actual (Perles Roselló, Sortino Barrionuevo y Cantanero Prados, 2017; Olcina Cantos y Oliva Cañizares, 2020).

En la sociedad actual, existe un alto grado de exposición a la peligrosidad, lo que lleva a la búsqueda de unas relaciones causales que, lejos de acercarse a las connotaciones de una naturaleza embrutecida, se aproxima más a la consideración de que las sociedades transforman su territorio, asumen nuevas dinámicas, y el medio natural se redimensiona como un espacio de riesgo (Olcina Cantos, 2009). Las normativas y disposiciones legales en torno al fenómeno de las inundaciones se han venido incrementando en los últimos años mediante un tratamiento más detallado, en aras a una mejor legislación territorial para prevenir la ocupación de espacios inundables (Olcina Cantos, 2010). Ello es fundamental dado que la vulnerabilidad sólo puede definirse mediante un proceso de construcción social explicado por las formas en las que los actores sociales usan y producen el territorio (Maldonado y Cóccaro, 2011).

Los usos agrarios, por ejemplo, pueden promover tendencias ligadas al estado del suelo que a la larga conducen a procesos de incremento de la vulnerabilidad y con ello del riesgo de inundación, como es el caso en Extremadura, donde la actividad agrícola ostenta la mayor parte de la superficie ocupada de las áreas con riesgo de inundación. Tales características se resumen en el sistema de drenaje del suelo, los métodos de labranza (compactación, orientación del arado...), la elección de los cultivos y sus patrones de explotación y las vertientes (Organización Meteorológica Mundial, 2007).

En la actualidad, cualquier modelo de desarrollo territorial futuro, sobre todo desde el marco de la sostenibilidad, es difícilmente entendible si no viene acompañado de un incremento del nivel de riesgo en la sociedad (Lavell, 1998). Los grupos humanos deben reducir el margen de vulnerabilidad ante el riesgo y, al tiempo, deben incrementar las capacidades para gestionarlos (Anderson y Woodrow, 1989).

\section{Conclusiones}

El riesgo y la condición de riesgo son elementos simbióticos entre lo natural y lo humano. Los factores del riesgo constituyen la base sobre la cual estas relaciones se producirán generando energía por defecto (capacidad de absorción y regeneración del sistema o sistema resiliente) o por exceso (cuando en 
las relaciones se produce energía que el sistema no es capaz de absorber y se genera la desestabilización de las relaciones y por tanto el desastre) (García Gómez, 2005).

En este contexto, cabe destacar que el río Guadiana y su cuenca es la que mayor riesgo presenta ante este tipo de fenómenos, con un 70\% de los eventos de crecidas e inundaciones registradas, frente al 30\% de los eventos registrados en la cuenca del Tajo. Las zonas de mayor concentración del riesgo de inundaciones en la región la constituyen las Vegas del Guadiana en los entornos de los ríos Ruecas, Gargáligas, Zújar y el propio Guadiana. En Extremadura existe un total de 31.408 ha con alto riesgo de inundación para un tiempo de recurrencia de 10 años, de las que el $87,7 \%$ pertenecen a la cuenca del Guadiana y el $12,4 \%$ a la del Tajo.

Se ha observado el hecho de que la superficie agrícola en regadío es una de las actividades principalmente expuesta al riesgo de inundación, con el impacto económico que ello deriva para una región como la extremeña. En este sentido, se ha constatado que el potencial impacto económico asociado al fenómeno de las inundaciones para esta actividad, concentra el 42,9\% de los daños económicos estimados para una probabilidad de 10 años y el 30\% para una probabilidad de 100 años.

Las inundaciones podrían tener un coste asociado en Extremadura de aproximadamente 570.000.000€ en el caso de las zonas del alto riesgo (10 años), y de casi 2.500.000.000 € para los casos de riesgo de inundación a 100 años.

Las inundaciones en Extremadura pueden colocar en una situación comprometida a una parte importante de la población, donde se han contabilizado hasta 6.726 habitantes potencialmente expuestos en zonas de alto riesgo, y hasta 20.200 en zonas de riesgo con frecuencia media u ocasional. La búsqueda del equilibrio, por tanto, se convierte en la pieza clave de las relaciones entre los elementos del sistema de riesgo (Kay y Shneider, 1992 cit. por Leff, 2004). Estudios, como los de Cid-Ortiz, Castro Correa y Rugiero de Souza (2012), sugieren que una de las variables más importantes a la hora de comprender la vulnerabilidad social es, pues, la resiliencia. Es por ello que los principios directores de toda política de adaptación y reducción de riesgos han de asumir el trinomio formado por la resistencia (aplicación de medidas estructurales para la protección contra fenómenos), la resiliencia (minimizar el riesgo sobre los elementos vulnerables del territorio, incluyendo las vidas y las propiedades) y la retirada (abandono de las zonas peligrosas) (Schmidt-Thomé y Greiving, 2009).

Por ello es necesario disponer de elementos de planificación y gestión que abarquen la complejidad territorial (López Martínez et al., 2016), desde un enfoque orientado a la multiplicidad de observación y que redunde en la capacidad para hacer frente a los retos planteados en las escalas territoriales, el enfoque de múltiple peligro, la gestión de la información, los indicadores, así como los destinatarios (Fleischhauer, Greiving y Wanczura, 2007). No obstante, se vuelve especialmente complicada la construcción de sistemas de información con datos comparables que sirvan de indicadores no sólo de la realidad territorial, sino que además resulte en un método eficaz para evaluación de los peligros que afectan al desarrollo regional (Schmidt-Thomé y Greiving, 2009).

La mejor forma de configurar una estrategia de adaptación al riesgo de inundación es promoviendo la formación e información de la población. A través de procesos orientados a la determinación de la percepción social del riesgo, se esclarecen cuáles son las carencias de la población en cuanto a conocimiento, así como la determinación de los perfiles socioeconómicos de los grupos afectados (Torró Segura, Camarasa Belmonte y Pitarch Garrido, 2019).

Es por ello que resulta de vital importancia otorgar una necesaria visibilidad a cada uno de los fenómenos con potencial de riesgo en la región a través de su contextualización territorial, que incluya, como se ha tratado de abordar en la presente investigación, aportes relacionados con su distribución geográfica y análisis socioeconómico, como base para el establecimiento de un marco de referencia que apoye el desarrollo de acciones concretas orientadas a la minimización del impacto potencial y el aumento de la percepción social del fenómeno.

\section{Referencias}

Anderson. M. \& Woodrow, P. (1989). Rising fron the Ashes: Development strategies in times of disaster. Colorado: Westview Press. Boulder.

Ayala Carcedo, F. J. y Olcina Cantos, J. (Coords.) (2002). Riesgos Naturales. Barcelona: Ariel Ciencia. 
Blas Morato, R. y Nieto Masot, A. (2008). Mapa de riesgos de incendios forestales en la provincia de Cáceres. En L. Hernández-Calvento y J. M. Parreño (Coords.), Tecnologías de la Información Geográfica para el Desarrollo Territorial (pp. 488-501). XII Congreso Nacional de Tecnologías de la Información Geográfica. Las Palmas de Gran Canaria: Servicio de Publicaciones y Difusión Científica de la ULPGC.

Blas Morato, R. (2009). Validación de la Localización de Incendios Mediante "Global Fire Email Alerts [Beta]" en Áreas con Clima Mediterráneo. Revista de Teledetección: Revista de la Asociación Española de Teledetección, (31), 64-68. Recuperado de http://www.aet.org.es/revistas/revista31/Numero31_5.pdf

Bustillos Ardaya, A., Evers, M. \& Ribbe, L. (2019). Participatory approaches for disaster risk governance? Exploring participatory mechanisms and mapping to close the communication gap between population living in flood risk areas and authorities in Nova Friburgo Municipality, RJ, Brazil. Land Use Policy, (88). https://doi.org/10.1016/j.landusepol.2019.104103

Calvo García-Tornel, F. (1997). Algunas cuestiones sobre la geografía de los riesgos. Scripta Nova, (10). Recuperado de http://www.ub.edu/geocrit/sn-10.htm

Camarasa Belmonte, A. M. (2002). Crecidas e Inundaciones. En F. J. Ayala Carcedo y J. Olcina Cantos (Coords.), Riesgos Naturales (pp. 859-877). Barcelona: Ariel.

Cid-Ortiz, G., Castro Correa, C. y Rugiero de Souza, V. (2012). Percepción del riesgo en relación con capacidades de autoprotección y autogestión como elementos relevantes en la reducción de la vulnerabilidad en la ciudad de La Serena. Revista Invi, 27,(75), 105-142. http://dx.doi.org/10.4067/ $\underline{\text { S0718-83582012000200004 }}$

Consorcio de Compensación de Seguros (CCS). (2016). Estadística Riesgos Extraordinarios. Serie 19712015. CCS. Madrid: CCS.

Directiva 2007/60/CE del Parlamento Europeo y del Consejo del 23 de octubre de 2007 relativa a la evaluación y gestión de los riesgos de inundación. Diario Oficial de la Unión Europea (D.O.U.E). (2007).

European Environment Agency (EEA). (1998). State of the Environment report $n^{o}$ 2. Denmark: EEA Publications.

Fernández Garrido, M. I. (2006). Los riesgos naturales en España y en la Unión Europea: incidencia y estrategias de actuación (Tesis Doctoral). Universidad de Cantabria. Santander.

Fleischhauer, M., Greiving, S. y Wanczura, S. (2007). Planificación territorial para la gestión de riesgos en Europa. Boletín de la AGE, (45), 49-78. Recuperado de http://age.ieg.csic.es/boletin/45/03planificacion.pdf

Frigerio, I. y De Amicis, M. (2016). Mapping social vulnerability to natural hazards in Italy: A suitable tool for risk mitigation strategies. Environmental Science \& Policy, (63), 187-196. https://doi.org/10.1016/j. envsci.2016.06.001

Gallegos Reina, A. y Perles Roselló, M. J. (2019). Relaciones entre los cambios en los usos del suelo y el incremento de los riesgos de inundabilidad y erosión: análisis diacrónico en la provincia de Málaga (1957-2007). Boletín de la Asociación de Geógrafos Españoles, (81), 1-38. https://doi.org/10.21138/ bage. 2740

García Gómez, A. (2005). Naturaleza, efectos y gestión de catástrofes de un sistema social. En Ruano Gómez, J. (coord.) Riesgos colectivos y situaciones de crisis: el desafío de la incertidumbre (pp. 107-126). La Coruña: Servicio de Publicaciones de la Universidad de La Coruña.

García Lucas, A. y Carrascal Tirado, J. (2007). Planes Periurbanos de Prevención de Incendios Forestales en la interfaz urbano forestal en la Comunidad Autónoma de Extremadura. Sevilla: Wildfire 2007, $4^{\mathrm{a}}$ Conferencia Internacional sobre Incendios Forestales.

Instituto Geográfico Nacional (IGN). Cartografía de Referencia a través del Centro Nacional de Información Geográfica (CNIG). Recuperado de http://centrodedescargas.cnig.es/CentroDescargas/ index.jsp

Juárez Sánchez-Rubio, C. (1978). Precipitación y dedicación agraria en la cuenca del Guadiana. Revista de Estudios Extremeños, 34(3), 593-625. Recuperado de https://www.dip-badajoz.es/cultura/ceex/reex digital/reex_XXXIV/1978/T.\%20XXXIV\%20n.\%203\%201978\%20sept.-dic/RV10674.pdf

Junta de Extremadura. (2006). Plan Territorial de Protección Civil de la Comunidad Autónoma de Extremadura (PLATERCAEX).

Junta de Extremadura. (2007). Plan Especial de Protección Civil de Riesgo de Inundaciones para la Comunidad Autónoma de Extremadura (INUNCAEX). 
Kay, J. \& Schneider, E. (1992). Thermodynamics and measures of ecosystem integrity. En D. H. McKenzic,, D. E. Hyatt y , V. J. McDonal (Eds.) Ecological Indicators, vol 1. (pp. 159-182). Proceedings of the International Symposium on Ecological Indicators. Florida: Elseviver. https://doi.org/10.1007/978-14615-4659-7_12

Lavell, A. (1998). Decison making and risk management. In Conference: Futhering Cooperation in Science and Technology for Caribbean Development. Port of Spain, Trinidad.

Leff, E. (2004). Racionalidad Ambiental: la reapropiación social de la naturaleza. Siglo XXI.

López Martínez, F, Pérez Morales, A. y Gil Guirado, S. (2016). In landscape management all of us have something to say. A holistic method for landscape Preservability evaluation in a Mediterranean region. Land use policy: The International Journal Covering All Aspects of Land Use, (51), 172-183. https://doi. org/10.1016/j.landusepol.2015.11.004

López Rodríguez, E. (2015). Los mapas temáticos de riesgos como elementos divulgativos y didácticos de concienciación social. En Nieto Masot, A. (Ed.), Aplicaciones TIG en el Análisis Territorial: transferencia a Universidad, Sector Público y Empresas (pp. 191-208). Cáceres: Servicio de Publicaciones de la Universidad de Extremadura.

López, F. (2015). Análisis de la eficacia institucional ante inundaciones en el municipio de Totana (Murcia). Investigaciones Geográficas, (63), 85-97. https://doi.org/10.14198/ingeo2015.63.06

López Rodríguez, E. (2017). Social Perception of Risk: the case of forest fires in Sierra de Gata. ARKEOS, 41. Vol. II. En Oosterbeek, L., Werlen, B. and Caron, L. (Eds.) Sustainability and Sociocultural Matrices. Transdisciplinary contributions for Cultural Integrated Landscape Management (pp. 89-101). Maçao: Instituto Terra e Memória.

López Rodríguez, E. (2019). Impacto social y percepción de los riesgos naturales en Extremadura (Tesis Doctoral). Universidad de Extremadura. Cáceres.

Maldonado, G. I. y Cóccaro, J. M. (2011). Esquema teórico para el estudio de la vulnerabilidad socio-territorial a las inundaciones en ámbitos rurales. Revista Geográfica Venezolana, 52(2), 81-100. Recuperado de http://www.saber.ula.ve/bitstream/handle/123456789/34422/articulo5. pdf;jsessionid=35A07B5919DC88FB5B66DBAB65752CAD? sequence $=1$

Mateos Rodríguez, A. B., Leco Berrocal, F. y López Rodríguez, E. (2015). Análisis y evaluación del impacto socioeconómico de los incendios forestales en Extremadura. En J. de la Riva, P. Ibarra, R. Montorio y M. Rodrigues, M. (Eds.), Análisis espacial y representación geográfica: innovación y aplicación (pp. 1765-1774). Zaragoza: Universidad de Zaragoza-Asociación de Geógrafos Españoles.

Mateos Rodríguez, A. B. y López Rodríguez, E. (2016). Desarrollo Rural y Riesgos Naturales en Extremadura. Instrumentos normativos y necesidades de actuación. En F. Leco (coord.), Territorio y Desarrollo Rural: aportaciones desde el ámbito investigador (pp. 87-108). Cáceres: Grupo de Estudios sobre Desarrollo Rural y Local en Espacios de Frontera. Universidad de Extremadura.

Ministerio para la Transición Ecológica y el Reto Demográfico (MITERD): Planes de Gestión del Riesgo de Inundación. Recuperado de https://www.MITERD.gob.es/es/agua/temas/gestion-de-los-riesgos-deinundacion/planes-gestion-riesgos-inundacion/default.aspx

Ministerio para la Transición Ecológica y el Reto Demográfico (MITERD): Cartografía del Área de actividad del Agua. Recuperado de https://www.MITERD.gob.es/es/cartografia-y-sig/ide/descargas/ agua/default.aspx

Morote Seguido, A.F. y Pérez Morales, A. (2019). La comprensión del riesgo de inundación a través del trabajo de campo: una experiencia didáctica en San Vicente del Raspeig (Alicante, España). Vegueta. Anuario de la Facultad de Geografía e Historia, (19), 609-631. Recuperado de http://revistavegueta. ulpgc.es/ojs/index.php/revistavegueta/article/view/470/699

Moya Ignacio, M., Mateos Vivas, J. J., Fortes Gallego, R., Ramírez Vera, A., Navero García, M. y Moreno Collado, F. L. (2009). Propuestas de Prevención de Incendio Forestal en la Interfaz Urbano-Forestal de 4 Municipios de Extremadura. Montes y Sociedad: Saber qué hacer. $5^{\text {a }}$ Congreso Forestal Español. Ávila: Junta de Castilla y León.

Navazo Arenas, G., Nieto Masot, A. y Moreno Marcos, G. (2016). Análisis de incendios forestales mediante Sistemas de Información Geográfica y Teledetección. Estudio de caso en Sierra de Gata (2015). En Nieto Masot, A. (Coord.), Tecnologías de la Información Geográfica en el Análisis Espacial. Aplicaciones en los Sectores Público, Empresarial y Universitario (pp. 247-268). Universidad de Extremadura. 
Grupo de Investigación en Desarrollo Territorial Sostenible y Planificación Territorial. Grupo de Investigación Geo-Ambiental.

Nieto Masot, A., García Paredes, C. y Fernández Sánchez, A. (2011). Estudio de casos para detección y análisis de fenómenos naturales con imágenes de SEVIRI, MODIS y LANDSAT TM 5: emisiones volcánicas, ciclogénesis explosivas y grandes incendios forestales. Geofocus, (11), 375-408. Recuperado de http://www.geofocus.org/index.php/geofocus/article/view/233

Olcina Cantos, J. (2008). Cambios en la consideración territorial, conceptual y de método de los riesgos naturales. Scripta Nova, XII, 270(24). Recuperado de http://www.ub.edu/geocrit/sn/sn-270/sn-270-24. $\underline{h t m}$

Olcina Cantos, J. (2009). España, territorio de riesgo. Enseñanzas de las Ciencias de la Tierra, 17(3), 242-253. Recuperado de http://hdl.handle.net/10045/23017

Olcina Cantos, J. (2010). El tratamiento de los riesgos naturales en la planificación territorial de escala regional. Papeles de Geografía, (51-52), 223-234. Recuperado de https://revistas.um.es/geografia/ article/view/114531

Olcina Cantos, J. y Oliva Cañizares, A. (2020). Medidas estructurales versus cartografía de inundación en la valoración del riesgo en áreas urbanas: El caso del barranco de las Ovejas (Alicante, España). Cuadernos Geográficos, 59(2), 199-220. https://doi.org/10.30827/cuadgeo.v59i2.10278

Organización Metereológica Mundial. (2007). The role of Land-Use Planning in flood management. A tool for Integrated Flood Management. Recuperado de http://www.apfm.info/pdf/ifm_tools/Tools_The_ Role_of_Land_Use_Planning_in_FM.pdf

Patricia Torchia, N. (2011). La Planificación Territorial y el Análisis del Riesgo de Desastres en el ámbito de la Inversión Pública. Desarrollo Local Sostenible, 4(11).

Perles Roselló, M. J., Sortino Barrionuevo, J. F. y Cantanero Prados, F. (2017). Cartografía de la vulnerabilidad del territorio frente al riesgo de inundación. Propuesta adaptada a la Directiva Europea de Inundaciones y normativas derivadas. Boletín de la Asociación de Geógrafos Españoles, (75), 341-372. https://doi.org/10.21138/bage.2504

Potenciano de las Heras, Á. (2004). Estudio de las Inundaciones Históricas del río Amarguillo (Toledo)(Tesis Doctoral). Universidad Complutense de Madrid. Madrid.

Pulido Díaz, F, Bertomeu García, M., del Pozo Barrón, J. L., Giménez Fernández, J. C. y Moreno Marcos, G. (2017). Elementos para un modelo integrado de prevención de incendios en la zona de alto riesgo de Gata-1 Hurdes (Cáceres). $7^{\circ}$ Congreso Forestal Español: Gestión del Monte: Servicios Ambientales y Bioeconomía.

Real Decreto 903/2010, de 9 de julio, de evaluación y gestión de riesgos de inundación. Boletín Oficial del Estado, 15 de julio de 2010, núm. 171.

Real Decreto Legislativo 1/2001, de 20 de julio, por el que se aprueba el texto refundido de la Ley de Aguas. Boletín Oficial del Estado, 24 de julio de 2001, núm. 176.

Ruiz Pérez, M. (2012). Vulnerabilidad territorial frente a desastres naturales: el caso de la isla de Mallorca (Baleares, España). Geofocus, (12), 16-52. Recuperado de http://www.geofocus.org/index.php/ geofocus/article/view/237

Saurí i Pujol, D. y Ribas Palom, A. (2006). De la geografía de los riesgos a las geografías de la vulnerabilidad. En Nogué i Font, J. y Romero, J. (coord.), Las otras geografías (pp. 285-300). Valencia: Tirant lo Blanch, col. Crónica.

Saurí, D., Ribas, A., Lara, A. y Pavón, D. (2010). La percepción del riesgo de inundación: experiencias de aprendizaje en la Costa Brava. Papeles de Geografía, (51-52), 269-278. Recuperado de https://revistas. um.es/geografia/article/view/114571

Schmidt Thomé, P. y Greiving, S. (2009). La respuesta a los peligros naturales y al cambio climático en Europa. Investigaciones Geográficas, (49), 23-49. https://doi.org/10.14198/INGEO2009.49.02

Torró Segura, M., Camarasa Belmonte, A. y Pitarch Garrido, M. D. (2019). Percepción del riesgo de inundación en el municipio de Ontiyent (Comunitat Valenciana). Cuadernos de Geografía, (103), 117-140. https://doi.org/10.7203/CGUV.103.16227

Van Alphen, J., Martini, F, Loat, R., Slomp, R. \& Passchier, R. (2009). Flood risk mapping in Europe, experiences and best practices. Journal of Flood Risk Management, (2), 285-292. https://doi. org/10.1111/j.1753-318X.2009.01045.x 